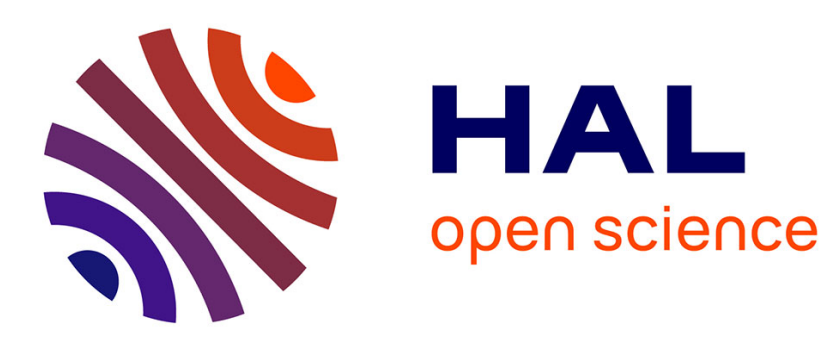

\title{
Complémentation énergétique de rations à base de foin pour les vaches laitières (1)
}

Agnès Hauwuy, J. Paradis, J.B. Coulon

\section{To cite this version:}

Agnès Hauwuy, J. Paradis, J.B. Coulon. Complémentation énergétique de rations à base de foin pour les vaches laitières (1). Productions Animales, 1992, 5 (5), pp.339-346. hal-00895989

\section{HAL Id: hal-00895989 \\ https://hal.science/hal-00895989}

Submitted on 1 Jan 1992

HAL is a multi-disciplinary open access archive for the deposit and dissemination of scientific research documents, whether they are published or not. The documents may come from teaching and research institutions in France or abroad, or from public or private research centers.
L'archive ouverte pluridisciplinaire HAL, est destinée au dépôt et à la diffusion de documents scientifiques de niveau recherche, publiés ou non, émanant des établissements d'enseignement et de recherche français ou étrangers, des laboratoires publics ou privés. 
INRA Prod. Anim., 1992, 5 (5), 339-346.
Agnès HAUWUY, J. PARADIS, J.B. COULON*

GIS-SUACI Alpes du Nord, 1 rue du Chateau, 73000 Chambéry

* INRA Laboratoire Adaptation des Herbivores aux Milieux

Theix, 63122 St Genès Champanelle

\section{Complémentation énergétique de rations à base de foin pour les vaches laitières}

Comparativement aux autres fourrages, il existe assez peu d'études récentes sur l'alimentation des vaches laitières avec des rations à base de foin, et celles qui sont disponibles concernent essentiellement des foins de prairies monospécifiques (Vinet et al 1980, Bertilsson et Burstedt 1983, Steacy et al 1983, Dulphy 1987, Kaiser et Combs 1989). Ce fourrage fait pourtant partie de la ration des vaches laitières dans de nombreuses régions et représente parfois l'unique fourrage distribué dans les zones de montagne (Franche-Comté, Alpes du Nord), où la transformation du lait en certains fromages (Comté, Beaufort, Reblochon) interdit l'utilisation d'ensilage d'herbe. Dans ces régions, des travaux importants ont récemment été entrepris pour mieux évaluer la diversité et les caractéristiques des prairies de fauche (Fleury et al 1988) et pour préciser la valeur nutritive et l'ingestibilité des foins qui en sont issus.

L'objectif de cette étude a été de comparer les performances de vaches laitières utilisant ces foins dans des conditions de complémentation plus ou moins favorables. Elle a été réalisée dans une exploitation des Alpes du Nord dont les principales caractéristiques sont précisées au tableau 1. Ceci a permis de disposer d'animaux (de race Abondance) et de foins représentatifs de cette région.

\section{Résumé}

Quarante-huit vaches laitières de race Abondance ont été utilisées au cours d'un essai conduit 2 années consécutives. Au cours de l'hiver tous les animaux recevaient à volonté une ration à base de foin de prairie permanente des Alpes du Nord. Chaque année, au cours des 16 premières semaines de lactation hivernale, 2 lots ont été constitués : les vaches du lot Haut étaient complémentées selon les recommandations, tandis que celles du lot Bas recevaient $2 \mathrm{~kg} / \mathrm{j}$ de concentré de moins. La composition de l'aliment concentré était ajustée de manière à ce que les apports azotés soient identiques dans les 2 lots. Les vaches du lot Bas n'ont produit que $0,5 \mathrm{~kg} / \mathrm{j}$ de lait de moins que celles du lot Haut, en raison d'un mobilisation de leurs réserves corporelles supérieure, mais leur taux protéique a été significativement plus faible (- $1 \mathrm{~g} / \mathrm{kg}$ ). La valeur d'encombrement des foins utilisés a été faible (comprise entre 1 et $1,05 \mathrm{UEL} / \mathrm{kg} \mathrm{MS}$ ), inférieure à celle attendue pour ce type de fourrage.

\section{1 / Conditions expérimentales}

\section{1 / Animaux et alimentation}

Chaque hiver, toutes les vaches vêlant en début de saison ( 7 décembre en moyenne) ont été utilisées, de manière à disposer d'au moins 16 semaines de lactation hivernale, soit au total 50 vaches en 2 ans (respectivement 22 et 28). Les vaches ont été conduites en stabulation entravée non paillée et traites en place 2 fois par jour.

Au cours de l'hiver (début novembre à mimai), tous les animaux ont reçu une ration composée de foin (distribué à volonté en 2 repas par jour, avant la traite) et de regain (2 à $6 \mathrm{~kg} / \mathrm{j}$ selon la qualité du foin, offerts en un repas, avant la distribution du foin du soir).

En fin de gestation et durant la première semaine de lactation, toutes les vaches ont été alimentées de la même manière. Elles ont reçu respectivement 1,2 et $3 \mathrm{~kg} / \mathrm{j}$ d'aliment concen-

(1) Etude réalisée dans le cadre du programme de RechercheDéveloppement des Alpes du Nord (SUACI, 1 rue du Chateau, 73000 Chambéryl. 
L'expérimentation s'est déroulée sur l'exploitation du GAEC du Tronchet, à Abondance en Haute-Savoie. Située à $950 \mathrm{~m}$ d'altitude, il s'agit d'une exploitation spécialisée en élevage bovin laitier, où le lait est transformé en fromage d'Abondance. Le troupeau est composé de 40 vaches laitières de race Abondance (produisant environ $4000 \mathrm{~kg} / \mathrm{an}$ ) et de 50 génisses qui assurent la totalité du renouvellement. Les vêlages ont lieu principalement entre les mois de novembre et mars ( $75 \%$ ). L'âge au premier vêlage est de 36 mois en moyenne. Les animaux sont logés au cours de la période hivernale (mi-novembre à mi-mai) en stabulation entravée et sont traits 2 fois par jour en place.

L'alimentation hivernale est exclusivement à base de foin et de regain. Ces fourrages proviennent de prairies naturelles ( 30 ha environ), dont la végétation se caractérise par une proportion non négligeable de dicotylédones exubérantes (Anthrisque et Géranium). Cette végétation résulte des conditions pédoclimatiques (milieu frais sans sécheresse estivale) et des pratiques agricoles (forte fertilisation organique sous forme de lisier et récolte des fourrages réalisée jusqu'à la fructification du Dactyle). Les fourrages sont récoltés en vrac et ventilés.

Pendant la période estivale, l'ensemble des animaux pâture sur 3 alpages, répartis entre 1200 et $1900 \mathrm{~m}$ d'altitude, afin de bénéficier de l'étagement du développement de la végétation. Les vaches laitières sont ramenées 2 fois par jour au châlet pour la traite. Elles ne reçoivent aucune complémentation au cours de cette période.

Sur cette exploitation, la main d'oeuvre est relativement importante ( $3 \mathrm{UTH}$ ), du fait de la transformation fromagère et de sa commercialisation d'une part et de l'utilisation d'estives par les vaches laitières d'autre part.

tré (céréales aplaties $(50 \%$ orge-50\% maïs) et $1 \mathrm{~kg} / \mathrm{j}$ de tourteau de soja) au cours des 3 dernières semaines précédant la date présumée de vêlage. En première semaine de lactation, la quantité de concentré offerte a été augmentée de $0,25 \mathrm{~kg}$ par jour.

A la fin de la première semaine de lactation, les vaches ont été réparties en 2 lots sur la base de leur rang de lactation, de leur date de vêlage, de leur niveau de production initiale (production moyenne des 4ème, 5ème et 6ème jours de la lactation en cours (PI)), et de leur poids vif (mesuré au cours de la lère semaine de lactation). Le premier lot (lot $\mathrm{H}$ ) a été complémenté suivant les recommandations INRA (Faverdin et al 1987), en retenant une persistance mensuelle de production au delà de la 6 ème semaine de lactation de $94 \%$ pour les primipares et de $89 \%$ pour les multipares. Les vaches du second lot (lot $B$ ) ont reçu, pour un même potentiel de production, $2 \mathrm{~kg} / \mathrm{j}$ de concentré en moins que celles du lot $\mathrm{H}$. Les quantités d'aliment concentré ont été prédéterminées individuellement pour tout l'hiver en fonction de la production maximale attendue des animaux (PM). La première année, celle-ci a été estimée à partir de la production initiale (PI), d'après les équations proposées par Faverdin et al (1987). Les résultats de la première année d'expérience ayant mis en évidence des accroissements de production (PM - PI) bien inférieurs à ceux attendus, la seconde année l'estimation de la production maximale a été réalisée en ajoutant, pour tous les animaux, $4 \mathrm{~kg}$ de lait à la production initiale. La proportion de tourteau de soja dans l'aliment concentré a été ajustée dans chaque lot de manière à ce que les apports azotés soient identiques dans les 2 lots et couvrent les besoins immédiatement après le vêlage. Elle a été en moyenne de respectivement 25 et $50 \%$ dans les lots $\mathrm{H}$ et $\mathrm{B}$. De manière à réaliser des apports nutritifs voisins au cours de la lacta- tion, et compte tenu des différents types de foin utilisés, les apports de tourteau de soja et la part de regain dans la ration ont été ajustés en fonction de la valeur nutritive des foins distribués. En pratique, ces modifications ont été peu nombreuses et de faible amplitude $(2 \mathrm{~kg} / \mathrm{j}$ de regain pendant 2 mois, et $-0,5 \mathrm{~kg} / \mathrm{j}$ de tourteau de soja pendant 10 jours). Un complément minéral (12 P-12 Ca) a été distribué à tous les animaux à raison de 100 à $300 \mathrm{~g} / \mathrm{j}$ selon leurs besoins. Les aliments concentrés ont été distribués en 2 repas par jour, après chaque distribution de foin. Les animaux disposaient en permanence d'eau et de pierre à sel.

La mise à l'herbe a eu lieu respectivement les 13 et 20 mai au cours des 2 années, après 3 jours de distribution d'herbe à l'auge. Au pâturage (en alpage), tous les animaux ont été conduits de la même façon, sans apport d'aliment complémentaire.

Les inséminations ont été réalisées sur chaleurs naturelles.

\section{$1.2 /$ Mesures}

Les quantités d'aliments offertes ont été pesées individuellement pour chaque type d'aliment 3 jours consécutifs par quinzaine. Les refus de foin ont été prélevés une fois par jour avant la distribution du foin du matin. Pour chaque lot de foin, ainsi que sur le regain et les refus une analyse de la composition chimique et une estimation de la digestibilité de la matière organique (méthode enzymatique) ont été réalisées. On en a déduit leur valeur nutritive (tableau 2 ).

Les quantités de lait produites ont été pesées individuellement, matin et soir, 3 jours par semaine. Les taux butyreux et protéique et la teneur en urée du lait ont été déterminés une fois par semaine, sur un échantillon 
Tableau 2. Composition chimique et valeur nutritive des aliments.

\begin{tabular}{|l|l|l|c|c|}
\hline & Foins (1) & Regain & Céréales $(2)$ & $\begin{array}{c}\text { Tourteau } \\
\text { de soja }\end{array}$ \\
\hline Matière sèche (\%) & $85(81-88)$ & 84 & 88 & 88 \\
Matière organique (\%MS) & $92(90-94)$ & 88 & 98 & 94 \\
MAT (\%MS) & $8,3(5,9-11,4)$ & 13,9 & 10,0 & 46,0 \\
CB (\%MS) & $34,3(29,1-38,5)$ & 24,7 & 3,4 & 4,3 \\
Phosphore (g/kg MS) & 2,4 & 2,8 & 3,2 & 6,7 \\
Calcium (g/kg MS) & 7,5 & 13,0 & 0,5 & 3,2 \\
Digestibilité MO (\%) & $58(51-65)$ & 69 & & 1,11 \\
UFL $(/ \mathrm{kg}$ MS) & $0,66(0,58-0,75)$ & 0,81 & 1,17 & 372 \\
PDIN (g/kg MS) & $51(39-71)$ & 88 & 80 & 277 \\
PDIE (g/kg MS) & $66(60-78)$ & 86 & 108 & \\
UEL (/kg MS) & 1,15 & 1,05 & & \\
\hline
\end{tabular}

(1) 7 lots de foin de 6 à 13 tonnes chacun ont été utilisés chaque année. Les valeurs des lots extrêmes sont précisées entre parenthèses.

(2) $50 \%$ d'orge et $50 \%$ de mais.

(3) mesurée par la méthode enzymatique à la pepsine cellulase.

(4) d'après les équations d'Andrieu et Demarquilly (1987).

(5) estimations ayant servi à l'élaboration du plan de rationnement.

moyen de 2 traites consécutives. Le $\mathrm{pH}$ du lait, sa teneur en calcium et son aptitude à la coagulation (temps et indice de coagulation) ont été mesurés individuellement 4 fois dans l'hiver et une fois en début de période de pâturage (la 2ème année seulement), sur un échantillon de la traite du matin.

Les vaches ont été pesées une fois au cours de la première semaine de lactation puis toutes les 2 semaines, en début d'après-midi. Leur état d'engraissement (noté de 0 à 5 , par observation visuelle, d'après la grille proposée par Bazin (1985) a été apprécié la semaine précédant le vêlage puis toutes les 4 semaines.

Les bilans énergétiques et azotés ont été calculés par différence entre les apports (corrigés de la valeur nutritive des refus qui tient compte du taux d'humidité et de la proportion de tiges et de brisures) et les besoins des animaux, estimés à partir de leur production laitière et de leur poids vif (Faverdin et al 1987). Les apports énergétiques ont été corrigés pour tenir compte des interactions digestives et métaboliques (Vermorel et al 1987).

\section{3 / Analyse des résultats}

L'analyse des résultats a été effectuée à l'échelle de l'hiver (16 premières semaines de lactation). Les données ont été traitées par analyse de variance en introduisant les facteurs lot, année, rang de lactation (primipare ou multipare) et la production initiale comme covariable. Les interactions lot*année, lot*rang de lactation et lot*PI n'ayant jamais été significatives, elles n'ont pas été introduites dans les analyses définitives.

\section{2 / Résultats}

Au cours des 2 années expérimentales, les mammites ont représenté le principal trouble sanitaire observé ( 8 cas). Deux d'entre elles, survenues dès la première semaine de lactation, ont conduit à l'élimination des animaux pour le traitement des données de la lère année. L'effectif final a donc été de 48 vaches.

\section{1 / Effet de l'année}

La production laitière des animaux a été significativement supérieure au cours du 2ème hiver $(+1,3 \mathrm{~kg} / \mathrm{j}$, soit $+8 \%, \mathrm{P}<0,01)$. Cet écart semble dû d'une part à un meilleur potentiel de production la seconde année (production initiale supérieure de $0,7 \mathrm{~kg} / \mathrm{j}$ ), lié en partie à une proportion de primipares moins importante $(29 \%$ contre $40 \%)$, d'autre part à une meilleure extériorisation de ce potentiel : l'augmentation de production en début de lactation a en effet été supérieure la seconde année $(+3,8 \mathrm{~kg} / \mathrm{j}$ entre PI et PM contre $+2,3$ $\mathrm{kg} / \mathrm{j}, \mathrm{P}<0,01)$. Cette différence peut s'expliquer par un état corporel au vêlage légèrement meilleur la seconde année $(+0,2$ point), mais surtout par une ingestion de fourrages bien supérieure en début de lactation (respectivement 10,6 et $12,1 \mathrm{~kg} \mathrm{MS} / \mathrm{j}$ au cours des 8 premières semaines, soit $+14 \%$ ), bien que la valeur nutritive des foins distribués à cette période ait été voisine les 2 années (respectivement 0,65 et $0,66 \mathrm{UFL} / \mathrm{kg}$ MS). En l'absence d'interaction année*traitement, les résultats seront présentés pour l'ensemble des 2 années.

\section{2 / Quantités ingérées}

Les quantités de fourrages ingérées ont augmenté plus fortement entre la 2ème et la 
16 ème semaine de lactation dans le lot $B$ que dans le lot $\mathrm{H}(+3,5 \mathrm{~kg} \mathrm{MS} / \mathrm{j}$ contre $+2,5)$ (figure 1). En moyenne sur cette période l'écart a été de + 0,5 kg MS/j $(12,4 \mathrm{~kg} \mathrm{MS} / \mathrm{J}$ contre 11,9 pour le lot $\mathrm{H})$. Il a été plus important en fin d'hiver qu'en début $(+0,8$ contre $+0,1 \mathrm{~kg}$ $\mathrm{MS} / \mathrm{j}$ )(tableau 3). L'écart d'apport de concentré ayant été constant au cours de l'hiver $(1,6 \mathrm{~kg}$ $\mathrm{MS} / \mathrm{j})$, le taux de substitution est donc passé de 0,1 en début de lactation à 0,4 au cours des semaines 9 à 16 .

Au cours de l'hiver, le concentré a représenté respectivement 29 et $21 \%$ de la ration totale dans les lots $\mathrm{H}$ et $\mathrm{B}$. Les quantités de refus ont été similaires pour les 2 lots $(1,3 \mathrm{~kg} \mathrm{MS} / \mathrm{j})$ et régulières au cours des 16 semaines expérimentales.

Entre la 9ème et la 16ème semaine de lactation, les quantités totales ingérées ont été de respectivement 17,0 et $16,1 \mathrm{~kg} \mathrm{MS} / \mathrm{j}$ dans les lots $\mathrm{H}$ et $\mathrm{B}$ (soit respectivement 2,75 et 2,65 $\mathrm{kg} / 100 \mathrm{~kg}$ poids vif). Elles ont augmenté avec le poids vif et la production laitière. Lorsque l'on tient compte de ces 2 variables, les quantités ingérées n'ont pas été différentes entre les primipares et les multipares et ont varié de 1,4 $\mathrm{kg} \mathrm{MS} / 100 \mathrm{~kg}$ poids vif et de $0,336 \mathrm{~kg} \mathrm{MS} / \mathrm{kg}$ de lait. Le passage d'un lot de foin à l'autre a entraîné des modifications sensibles des per- formances des animaux qui seront détaillées par ailleurs (Hauwuy et al 1993).

Figure 1. Evolution des quantités ingérées.

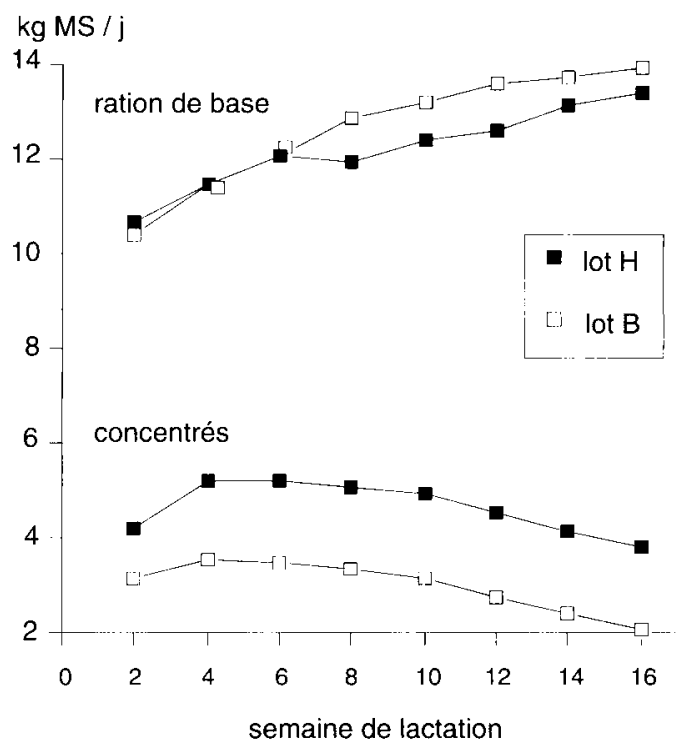

Tableau 3. Performances hivernales des animaux.

\begin{tabular}{|c|c|c|c|c|}
\hline Lot & Haut & Bas & & ETR $^{(1)}$ \\
\hline Effectif & 23 & 25 & & \\
\hline Date de vêlage & $7 / 12$ & $7 / 12$ & & \\
\hline Poids au vêlage & 618 & 608 & & \\
\hline Etat au vêlage & 3,4 & 3,5 & & \\
\hline Production initiale $(\mathrm{kg} / \mathrm{j})$ & 16,7 & 16,6 & & \\
\hline \multicolumn{5}{|l|}{ Semaines 2 à 8} \\
\hline Production maximale $(\mathrm{kg} / \mathrm{j})$ & 19,4 & 19,7 & NS & 1,4 \\
\hline Lait $(\mathrm{kg} / \mathrm{j})$ & 18,1 & 18,3 & NS & 1,6 \\
\hline Taux butyreux (g/kg) & 34,5 & 35,2 & NS & 2,4 \\
\hline Taux protéique $(\mathrm{g} / \mathrm{kg})$ & 29,9 & 29,2 & NS & 1,5 \\
\hline Concentrés (kg MS/j) & 5,1 & 3,6 & $* *$ & 0,5 \\
\hline Fourrages (kg MS/j) & 11,3 & 11,5 & NS & 1,1 \\
\hline Bilan énergétique (UFL/j) & 0,8 & $-0,4$ & $* *$ & 1,1 \\
\hline Bilan azoté (g PDI/j) & 272 & 245 & NS & 114 \\
\hline \multicolumn{5}{|l|}{ Semaines 9 à $16^{2}$} \\
\hline Lait $(\mathrm{kg} / \mathrm{j})$ & 16,3 & 15,5 & NS & 1,6 \\
\hline Taux butyreux $(\mathrm{g} / \mathrm{kg})$ & 34,6 & 34,2 & NS & 2,6 \\
\hline Taux protéique $(\mathrm{g} / \mathrm{kg})$ & 30,8 & 29,4 & $* *$ & 1,6 \\
\hline Concentrés (kg MS/j) & 4,5 & 3,0 & $* *$ & 0,5 \\
\hline Fourrages $(\mathrm{kg} \mathrm{MS} / \mathrm{j})$ & 12,5 & 13,1 & NS & 1,3 \\
\hline Bilan énergétique $(\mathrm{UFL} / \mathrm{j})$ & 1,8 & 1,1 & $*$ & 1,1 \\
\hline Bilan azoté (g PDI/j) & 285 & 320 & NS & 95 \\
\hline Persistance & 0,985 & 0,977 & $* *$ & 0,09 \\
\hline \multicolumn{5}{|c|}{ Variations entre les semaines 1 et 16} \\
\hline Poids vif vide $(\mathrm{kg})$ & +4 & -11 & $*$ & 27 \\
\hline Etat corporel & $-0,3$ & $-0,7$ & $* *$ & 0,5 \\
\hline
\end{tabular}

NS : non significatif, $*: \mathrm{P}<0,05,{ }^{* *}: \mathrm{P}<0,01$

(1) écart type résiduel

(2) valeurs ajustées par analyse de variance

(3) persistance hebdomadaire, calculée entre les semaines 5 et 15 


\section{3 / Production et composition du lait}

Dans les 2 lots, le pic de production laitière a été observé en moyenne dès la 4ème semaine de lactation (figure 2) et l'augmentation entre la production initiale et la production maximale (PM-PI) a été voisine dans les 2 lots $(+2,9$ $\mathrm{kg}$ de lait/j) mais inférieure aux prévisions qui ont servi pour établir le rationnement des animaux (cf. Conditions expérimentales). Ainsi l'écart entre la production maximale observée et la production maximale prévue à partir des équations de Faverdin et al (1987) a été de - 3,6 kg/j pour les primipares et de - $2,5 \mathrm{~kg} / \mathrm{j}$ pour les multipares. Cet écart n'a pas dépendu de la production initiale.

Sur l'ensemble de la période expérimentale, l'écart de production laitière entre les lots $\mathrm{H}$ et B a été faible $(0,5 \mathrm{~kg} / \mathrm{j}$, soit $52 \mathrm{~kg}$ au total). Il a été très faible au cours des 8 premières semaines de lactation, puis s'est accru pour atteindre $1,2 \mathrm{~kg} / \mathrm{j}$ en 16 ème semaine de lactation, en raison d'une persistance de la produc-

Figure 2. Evolution de la production laitière et de la composition du lait au cours de l'hiver.

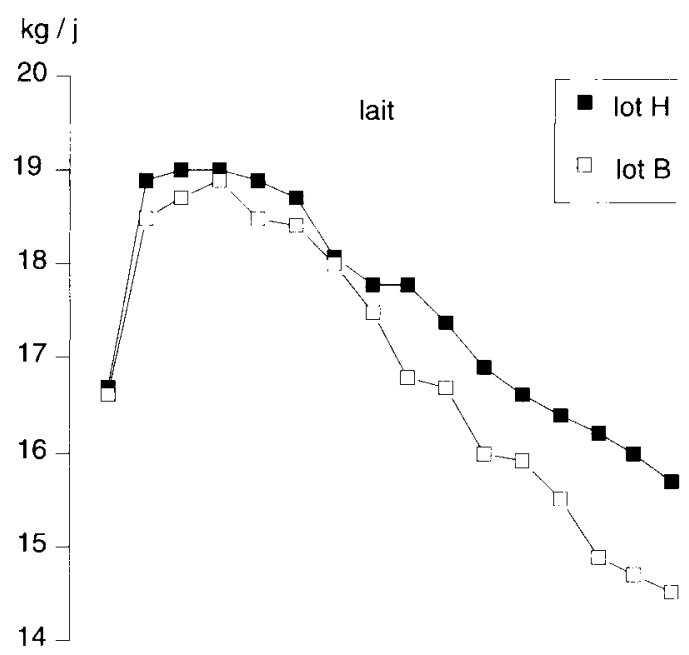

$\mathrm{g} / \mathrm{kg}$

38

36

34

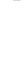

3

30

28

0

L.] taux butyreux

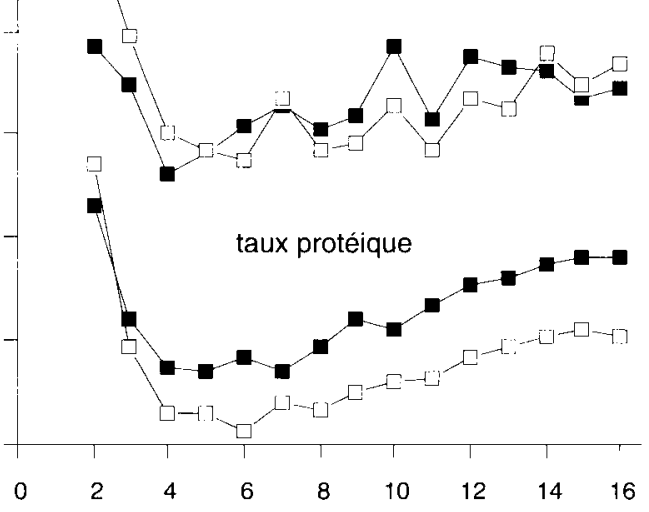

semaine de lactation tion laitière supérieure dans le lot $\mathrm{H}(0,985$ contre $0,977, \mathrm{P}<0,01)($ tableau 3 ). En conséquence, l'efficacité du concentré a été relativement faible sur l'ensemble de la période $(+0,3$ $\mathrm{kg}$ de lait par $\mathrm{kg}$ de concentré supplémentaire) et a augmenté avec l'avancement de la lactation : $+0,1$ et $+0,5$ respectivement pour la 1ère et 2ème période. Au cours de la 2ème période expérimentale, les animaux du lot $\mathrm{H}$ ont disposé de 1,4 UFL/j de plus que ceux du lot $B$. Le rendement marginal a donc été de 0,7 $\mathrm{kg}$ de lait/j par UFL supplémentaire. Ce rendement a été moins élevé chez les primipares que chez les multipares $(0,6$ contre $0,9 \mathrm{~kg} / \mathrm{UFL})$; chez ces dernières, il a augmenté avec le niveau de production des animaux (respectivement 0,7 et $1 \mathrm{~kg} / \mathrm{UFL}$ pour les multipares ayant une production initiale inférieure à 18 $\mathrm{kg} / \mathrm{j}$ et celles ayant une production initiale supérieure à $18 \mathrm{~kg} / \mathrm{j}$ ).

En moyenne, le niveau d'alimentation énergétique n'a pas eu d'effet significatif sur le taux butyreux, quelle que soit la période considérée (tableau 3). Par contre, le taux protéique a été significativement plus faible pour les vaches du lot $B$ que pour celles du lot $H(-1,0$ $\mathrm{g} / \mathrm{kg}, \mathrm{P}<0,05)$. L'écart s'est accru avec l'avancement de la lactation, atteignant $1,5 \mathrm{~g} / \mathrm{kg}$ en 16 ème semaine de lactation (figure 2). Compte tenu de ces évolutions, la production de matières grasses a été voisine dans les 2 lots, et la production de matières protéiques plus élevée dans le lot $\mathrm{H}(+30 \mathrm{~g} / \mathrm{j}$ comparativement au lot $\mathrm{B}, \mathrm{P}<0,05)$. Le taux de cellules du lait a été régulièrement faible (inférieur à $200000 / \mathrm{ml}$ ) et similaire entre les 2 lots. En moyenne au cours de l'hiver le taux de calcium du lait est resté relativement stable et voisin dans les 2 lots (respectivement 1,18 et 1,17 $\mathrm{mg} / \mathrm{l}$ dans les lots $\mathrm{H}$ et $\mathrm{B}$ ). Le $\mathrm{pH}$ du lait et son temps de coagulation ont été semblables dans les 2 lots ; ils ont augmenté régulièrement au cours de l'hiver (respectivement $+0,05$ et +10 min entre les mois de janvier et d'avril), vraisemblablement sous l'effet de l'avancement de la lactation.

La mise à l'herbe a entraîné une amélioration de la production laitière, plus importante dans le lot $\mathrm{B}$ que dans le lot $\mathrm{H}$ ( $\mathrm{de}+0,9 \mathrm{~kg} / \mathrm{j}$, entre les 2 dernières semaines hivernales et les 3 premières semaines de pâturage) (figure 3). L'augmentation du taux protéique a été importante dans les 2 lots, mais supérieure dans le lot $\mathrm{B}(+2,2 \mathrm{~g} / \mathrm{kg}$ contre $+1,6$ pour le lot $\mathrm{H})$. Après 8 semaines de pâturage, les taux protéiques des 2 lots étaient voisins et largement supérieurs à ceux observés en fin d'hiver. Le taux butyreux a augmenté très fortement à la mise à l'herbe (respectivement $+5,3$ et $+3,6$ $\mathrm{g} / \mathrm{kg}$ dans les lots $\mathrm{H}$ et $\mathrm{B}$ ), mais de manière temporaire, surtout dans le lot $B$, où 8 semaines après la mise à l'herbe le taux butyreux a été voisin de celui observé en fin de période hivernale (figure 3). D'autre part, la mise à l'herbe s'est accompagnée d'une augmentation très forte de la teneur en urée du lait $++355 \mathrm{mg} / \mathrm{l}$ entre les 2 dernières semaines hivernales et la première semaine de pâtura-

\section{En milieu de lactation, la production laitière a été augmentée de $0,7 \mathrm{~kg} / \mathrm{j}$ par $U F L$ supplémentaire, et le taux protéique de $1 \mathrm{~g} / \mathrm{kg}$.}


Figure 3. Evolution de la production laitière et de la composition du lait à la mise à l'herbe.

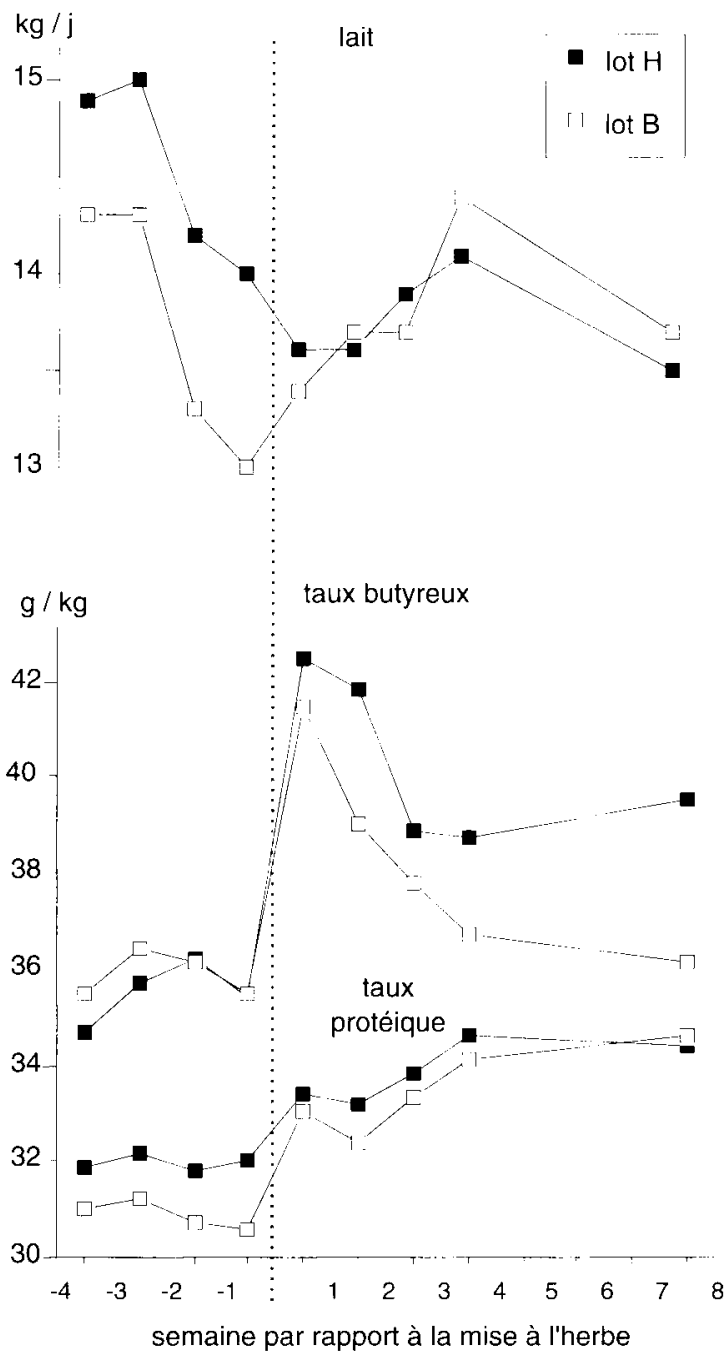

ge), mais de courte durée. Le $\mathrm{pH}$ du lait et sa teneur en calcium ont augmenté fortement, de manière identique dans les 2 lots (respectivement $+0,04$ et $+0,10 \mathrm{~g} / 1$ ) alors que le temps de coagulation et la teneur en phosphore ont diminué (respectivement - 7 min et - 0,21 g/l).

\section{4 / Poids vif et état corporel}

Au cours de la période hivernale, les variations de poids vif ont été faibles (figure 4). Les animaux du lot $B$ ont cependant perdu plus de poids que ceux du lot $\mathrm{H}$. La différence atteint $15 \mathrm{~kg}(\mathrm{P}<0,05)$ lorsque l'on corrige les poids vif pour tenir compte de l'augmentation du contenu digestif en début de lactation (Chilliard et al 1987) (tableau 3).

Parallèlement, les pertes d'état corporel ont duré plus longtemps et ont été supérieures dans le lot B (- 0,7 point entre les semaines 1 et 16 contre $-0,3$ point pour le lot $\mathrm{H}, \mathrm{P}<0,01$ ) (tableau 3).
Figure 4. Evolution du poids vif et des bilans énergétiques et azotés.

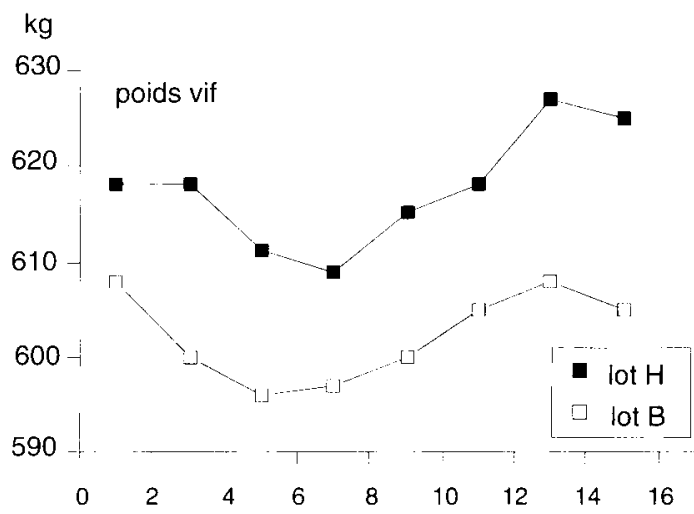

UFL / j

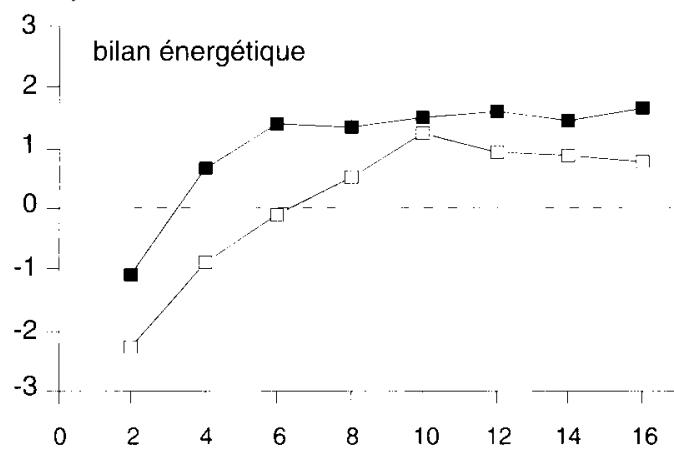

$\mathrm{gPDI} / \mathrm{j}$

400

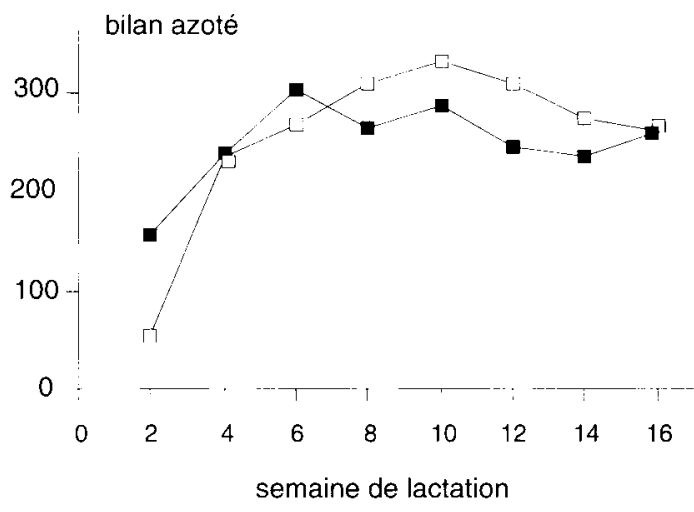

\section{5 / Bilans énergétiques et azotés}

Les bilans énergétiques ont été excédentaires dès la 4ème semaine de lactation pour les vaches du lot $\mathrm{H}$ et dès la 8ème pour celles du lot B (figure 4). A l'échelle de l'hiver, ils ont été significativement $(P<0,01)$ plus élevés pour le lot $\mathrm{H}(+1,2 \mathrm{UFL} / \mathrm{j}$ contre $+0,2$ pour le lot $\mathrm{B})$. Les bilans azotés ont été largement positifs quels que soient le lot et la période considérés. Ces excès d'azote se sont accompagnés de concentrations élevées d'urée dans le lait, en particulier dans le lot B (381 mg/l contre $323, \mathrm{P}<0,01$ ). 


\section{3 / Discussion}

La réponse des animaux à une modification des apports énergétiques a été conforme à celle généralement observée, compte tenu du niveau de couverture des besoins, élevés même dans le lot B, du fait de la surestimation du potentiel des animaux et de la sous-estimation de l'ingestibilité des fourrages. Cette situation explique que les pertes de poids vif et d'état corporel ont été limitées, malgré un bon état corporel au vêlage. En milieu de lactation (semaine 9 à 16), les réponses en lait ont été tout à fait comparables aux prévisions obtenues à partir des équations proposées par Coulon et Rémond (1991) $(0,7 \mathrm{~kg}$ lait/UFL contre 0,5$)$. La réponse du taux protéique a par contre été sensiblement supérieure à ces prévisions $(1 \mathrm{~g} / \mathrm{kg} / \mathrm{UFL}$ contre 0,6$)$. Cette réponse importante du taux protéique à un apport énergétique supplémentaire, même lorsque la couverture des besoins semble déjà largement réalisée, est particulièrement intéressante dans des situations comme celles des Alpes du Nord, où la transformation fromagère est la valorisation principale du lait. Cette réponse a été confirmée lors de la mise à l'herbe qui s'est accompagnée, même dans le lot $\mathrm{H}$, d'une augmentation sensible et durable du taux protéique, comme cela est couramment observé en zone herbagère de demi-montagne (Coulon $e t$ al 1986, Coulon et al 1988). Ces résultats montrent que dans les conditions de cette étude (stade de lactation avancé à la mise à l'herbe, alpage de faible altitude $(1200 \mathrm{~m})$ ), la mise à l'herbe s'accompagne d'une amélioration sensible des performances, contrairement à ce qui a pu être observé dans d'autres situations de montagne plus extrêmes (alpage à $2000 \mathrm{~m}$, niveau de production élevé à la mise à l'herbe : Zemp et al 1989).

Les quantités totales ingérées par les vaches de race Abondance sont élevées. Les valeurs observées sont en effet supérieures à la plupart de celles obtenues dans des conditions similaires (rations majoritairement à base d'herbe conservée, distribuée à volonté à des animaux d'un niveau de production modéré) par différents auteurs (Oldenbroek et al 1980, Krohn et al 1983, Gruber et al 1991, Pricaz 1991). Si l'on fait l'hypothèse que ces vaches ont une capacité d'ingestion voisine de celle des vaches Holstein et Montbéliardes qui ont servi à élaborer le système des unités d'encombrement, ces ingestions importantes conduisent à attribuer une ingestibilité très élevée aux fourrages utilisés (chez les multipares, on obtient ainsi des valeurs d'encombrement comprises entre 1 et 1,05 pour les foins utilisés en pleine lactation), bien supérieure à celles retenues pour ces types de foin (INRA, 1988). Ces valeurs sont certainement en partie dues à la distribution très libérale du foin (refus systématiquement supérieurs ou égaux à $10 \%$ ) qui a pu permettre aux animaux de réaliser un tri important conduisant en particulier à la non-consommation des tiges de Dicotylédones, qui pénalisent généralement l'ingestion de ces fourrages. Ces résultats seront développés par ailleurs (Hauwuy et al 1993).

Si les animaux de race Abondance semblent, dans leur réponse à une sous-alimentation énergétique, se comporter de manière identique à ceux d'autres races laitières françaises (Pricaz 1991), cette étude a cependant mis en évidence certaines particularités : d'une part l'accroissement de la production laitière observé en début de lactation a été faible, inférieur aux prévisions obtenues à partir des équations proposées par Faverdin et al (1987); d'autre part la capacité d'ingestion des primipares a été élevée ; exprimée en UEL, elle a été égale à $94 \%$ de celle des multipares lorsque l'on tient compte du poids vif et de la production laitière. Cette valeur, obtenue sur un faible nombre d'animaux, est légèrement supérieure à celle proposée par Faverdin et al (1987), élaborée il est vrai à partir de données concernant principalement des primipares vêlant à 2 ans, ce qui n'est pas le cas dans cet essai.

Les résultats de cette étude témoignent de la faisabilité d'un tel essai en ferme ; ils montrent que, moyennant une pression de mesure suffisante, il est possible d'obtenir dans ces conditions une précision acceptable des données : les écart types résiduels obtenus ont été voisins de ceux observés en domaine expérimental pour des essais du même type. Cela permet par ailleurs d'étudier ou de mettre en évidence des facteurs de variations du milieu spécifiques, difficilement appréhendables ailleurs. C'est le cas de la nature des foins utilisés dans ces zones de montagne et du type d'animal, dont les particularités doivent cependant faire l'objet de confirmation à plus grande échelle avant d'être pris en compte dans la conduite pratique des troupeaux.

\section{Remerciements}

Nous tenons à remercier Monsieur et Madame B. Folliet et Monsieur et Madame M. Girard-Derpraulet, qui ont mis leur exploitation à notre disposition et ont supporté patiemment les contraintes de l'expérimentation.

\section{Références bibliographiques}

Andrieu J., Demarquilly C., 1987. Valeur nutritive des fourrages : tables et prévision. Bull Tech. CRZV Theix, INRA, 70, 61-74.

Bazin S., 1985. Grille de notation de l'état d'engraissement des vaches Pie-Noires. Ed. ITEB, 149 rue de Bercy, 75595 Paris Cedex 12, 31 pp.

Bertilsson J., Burstedt E., 1983. Effects of conservation method and stage of maturity upon the feeding value of forages to dairy cows. 1. Forage intake and effects of concentrate/forage ratio. Swedish J. Agric. Res., 13, 189-200.

\section{Les valeurs \\ d'encombrement \\ des foins \\ ont été comprises \\ entre \\ 1 et 1,05 UEL/kg MS. \\ Ces valeurs \\ sont inférieures \\ à celles \\ habituellement \\ retenues pour ces \\ types de foins.}


Chilliard Y., Rémond B., Agabriel J., Robelin J., Vérité R., 1987. Variations du contenu digestif et des réserves corporelles au cours du cycle gestationlactation. Bull. Tech. CRZV Theix, INRA, 70, 117132.

Coulon J.B., Rémond B., 1991. Réponses de la production et de la composition du lait de vache aux variations d'apports nutritifs. INRA Prod. Anim., 4, 49-56.

Coulon J.B., Garel J.P., Hoden A., 1986. Evolution de la production et de la composition du lait à la mise à l'herbe. Bull. Tech. CRZV Theix, INRA, 66, 23-29.

Coulon J.B., D'Hour P., Petit M., 1988. Influence of transition feeding pattern on milk production at the turnout of cows to pasture. Livest. Prod. Sci., 20 , 119-134.

Dulphy J.P., 1987. Utilisation des foins par les vaches laitières. in : C. Demarquilly Ed., Les fourrages secs : récolte, traitement, utilisation. INRA Publications, Paris. 335-360.

Faverdin P, Hoden A., Coulon J.B., 1987. Recommandations alimentaires pour les vaches laitières. Bull. Tech. CRZV Theix, INRA, 70, 133152.

Fleury P., Jeannin B, Dorioz J.M., 1988. Typologie des prairies de fauche de montagne des Alpes du Nord humides. INRA, SUACI Montagne, rue du chateau, 73000 Chambéry, $130 \mathrm{pp}$.

Gruber L., Steinwender R., Krimberger K., Solkner J., 1991. Roughage intake of Simmental, Brown Swiss and Holstein Friesan cows fed rations with 0,25 and $50 \%$ concentrates. Livest. Prod. Sci., 27, 123-126.

Hauwuy A., Coulon J.B., Paradis J., 1993. Effet de la nature du foin sur les performances des vaches laitières. A paraître.
INRA, 1988. Alimentation des bovins, ovins et caprins. ED R. Jarrige, INRA Publications.

Kaiser R.M., Combs D.K., 1989. Utilization of three maturities of alfalfa by dairy cows fed rations that contain similar concentrations of fiber. J. Dairy Sci., $72,2301-2307$.

Krohn C.C., Hvelplund T., Andersen P.E., 1983. The effect on performance of different energy concentrations in complete rations for first lactation cows before and after calving. Livest. Prod. Sci., 10, 223-237.

Oldenbroek J.K., Van Eldik P., 1980. Differences in feed intake between Holstein Friesan, Dutch Red and White and Dutch Friesan cattle. Livest. Prod. Sci., 7, 13-23.

Pricaz F., 1991. Réactions des vaches laitières à des conditions de milieu difficiles : aptitude comparée de Tarines, Montbéliardes et Pie Noires en première et deuxième lactation. Mémoire de fin d'études. ENSA Rennes, 65 rue de St- Brieuc, 35042 Rennes.

Steacy G.M., Christensen D.A., Cochran M.I. Horton J., 1983. An evaluation of three stages of matutity of hay fed with two concentrate levels for lactating dairy cows. Can. J. Anim. Sci., 63, 623-629.

Vermorel M., Coulon J.B., Journet M., 1987. Révision du système des unités fourragères (UF). Bull. Tech. CRZV Theix, INRA, 70, 9-18.

Vinet C., Bouchard R., Saint Laurent G.S., 1980. Effects of stage of maturity of thimothy hay and concentrate supplementation on performance of lactating dairy cows. Can. J. Anim. Sci., 60, 511-521.

Zemp M., Leuenberger H., Kunzi N., Blum J.W., 1989. Influence of high altitude grazing on productive and physiological traits of dairy cows. 1 . Influence on milk production and body weight. J. Anim. Breed. Genet., 106, 278-288.

\section{Summary}

Effect of energy supply on performances of dairy cows fed with hay-based ration.

A total of 48 dairy cows (Abondance breed) were used in a 2-year experiment. The experiment was designed to examine the effect of concentrate levels during the winter period in addition to hay from native mountain grassland (Northen Alpes) offered ad libitum. Treatment $\mathrm{H}$ received concentrate at a high level and treatment $L$ at a low level $(2 \mathrm{~kg} / \mathrm{j}$ less than treatment $\mathrm{H})$. The composition of the concentate was adjusted to allow the same nitrogen supply for the 2 treatments. During winter (weeks 1-16 of lactation) milk production of treatments $H$ and $L$ were similar $(17.2 \mathrm{~kg} / \mathrm{j}$ vs. 16.7 $\mathrm{kg} / \mathrm{j})$. Protein content was higher $(+1 \mathrm{~g} / \mathrm{kg}, \mathrm{P}<$ 0.01 ) in treatment $H$. Body reserves losses were higher in treatment $B$. The fill value of hay was low (between 1 and $1.05 \mathrm{UEL} / \mathrm{kg} \mathrm{MS}$ ).

HAUWUY Agnès, PARADIS J., COULON J.B., 1992. Complémentation énergétique de rations à base de foin pour les vaches laitières. INRA Prod. Anim., 5 (5), 339346. 